A N N A L E S Annales de Bretagne et des Pays de l'Ouest

Anjou. Maine. Poitou-Charente. Touraine

111-1 | 2004

Varia

\title{
Vivre et écrire l'espace à Landévennec au IXe siècle
}

\section{Yves Morice}

\section{Q OpenEdition \\ Journals}

Édition électronique

URL : http://journals.openedition.org/abpo/1295

DOI : $10.4000 / a b p o .1295$

ISBN : 978-2-7535-1493-5

ISSN : 2108-6443

Éditeur

Presses universitaires de Rennes

Édition imprimée

Date de publication : 20 mars 2004

Pagination : 7-23

ISBN : 978-2-86847-976-1

ISSN : 0399-0826

\section{Référence électronique}

Yves Morice, "Vivre et écrire l'espace à Landévennec au IXe siècle », Annales de Bretagne et des Pays de l'Ouest [En ligne], 111-1 | 2004, mis en ligne le 20 mars 2006, consulté le 26 avril 2019. URL : http:// journals.openedition.org/abpo/1295; DOI : 10.4000/abpo.1295 


\title{
Vivre et écrire l'espace à Landévennec au IX ${ }^{\mathrm{e}}$ siècle
}

\author{
Yves MorICE \\ doctorant \\ CRHISCO - Université Rennes 2 Haute-Bretagne
}

Depuis sa fondation au cours du vi ${ }^{\mathrm{e}}$ siècle, l'abbaye finistérienne de Landévennec a connu par deux fois l'abandon. Comme pour beaucoup d'autres établissements monastiques, sa première ruine survient au cours de l'interrègne scandinave en Bretagne, sans doute peu avant 913, date pour laquelle une main porte cette mention en marge d'un calendrier de Landévennec conservé à Copenhague : " eodem anno destructum est monasterium sancti Uuinualoei a normannis ${ }^{1}$ ". Les moines en exil se réfugièrent en Neustrie, à Montreuil-sur-Mer, dont ils ne revinrent pas avant 936, date d'une importante donation du Duc Alain Barbetorte en leur faveur ${ }^{2}$.

Si après ces graves tribulations l'abbaye eut du mal à se relever et ne retrouva plus jamais sa gloire passée, la répétition d'une telle infortune lui fut cependant épargnée pendant plusieurs siècles, jusqu'à l'avènement d'un autre grand séisme, la Révolution. En 1790, les quatre moines - seulement! demeurant encore à Landévennec quittèrent alors les lieux dont tout laisse à penser qu'ils étaient déjà dans un état avancé de dégradation ${ }^{3}$. Cette foisci, il fallut attendre les années soixante pour que des moines bénédictins, des mauristes, viennent se réinstaller à cet endroit ${ }^{4}$, choisissant d'occuper la partie supérieure du vallon et laissant les ruines de l'ancienne abbaye, essentiellement l'abbatiale romane et une partie des murs du cloître moderne, en contrebas.

Cette pérennité du site, malgré ces évènements qui à chaque fois ne manquèrent que de peu d'être fatals, frappe les esprits et interroge l'historien. Cette constance est spectaculairement matérialisée par la strati-

1. Copenhague Thott 239. Cf. Deuffic, Jean-Luc, "Les manuscrits de Landévennec ", dans Simon, Marc, L'abbaye de Landévennec de saint Guénolé à nos jours, Rennes, OuestFrance, 1985, p. 272-274.

2. Cartulaire de Landévennec, 25, Le Men, Lucien et ERnault, Émile (éd.), Cartulaire de Landévennec, Mélanges Historiques, t. V, Paris, 1895, p. 562-563, ci-dessous : CL.

3. Simon, Marc, L'abbaye de Landévennec..., op. cit., p. 149-151.

4. Ibid., p. 167-168. 
graphie complexe qui témoigne des remaniements successifs du monastère au cours des siècles ${ }^{5}$. Si cette superposition étonnante fait à la fois tout l'intérêt et la difficulté du site, elle vient confirmer l'attachement extrême de la communauté à un emplacement bien précis, sur lequel on rebâtit encore et encore, et ce malgré les destructions ponctuelles, malgré l'exil, malgré l'humidité permanente due à l'épanchement de l'ancien ruisseau en nappe phréatique après l'attaque normande, qui rend les fondations instables et contraint à remblayer constamment afin de stabiliser les sols, rehaussés donc au fur et à mesure.

Pourquoi s'acharner? Certes l'endroit est charmant, niché dans une ria de l'Aulne, mais l'argument esthétique paraît forcément un peu léger lorsqu'on le met en balance avec tous les inconvénients. Un début de réponse est peut-être fourni par l'autre source majeure pour l'histoire de l'abbaye médiévale, le cartulaire de Landévennec. En effet, au retour de l'exil neustrien, la petite communauté s'est retrouvée devant la lourde tâche de réorganiser sa vie à Landévennec. Cela passe bien entendu par une reconstruction matérielle, mais aussi par la recomposition et la remise à plat du patrimoine foncier. C'est dans ce but qu'un scribe anonyme a compilé au milieu du $\mathrm{XI}^{\mathrm{e}}$ siècle cet ensemble de chartes et de notices. En tête de ce document, il recopie dans son intégralité le dossier hagiographique de saint Guénolé, le fondateur légendaire de l'abbaye : une grande vita prosimétrique ainsi que son abrégé, une homélie en douze leçons, composées l'une et l'autre par l'abbé Gurdisten; une hymne biographique alphabétique due au moine Clément; deux hymnes anonymes enfin, qui datent vraisemblablement de la seconde moitié du IX ${ }^{\mathrm{e}}$ siècle, tout comme les autres pièces ${ }^{6}$.

Ce choix de reprendre au XI ${ }^{\mathrm{e}}$ siècle des textes du $\mathrm{IX}^{\mathrm{e}}$ siècle sans les remanier ne laisse pas de surprendre. À ce titre, Landévennec semble réellement faire exception au sein d'une hagiographie bretonne préromane qui a plutôt tendance à abréger et à simplifier ses vieux textes, inclination récemment soulignée par Joseph-Claude Poulin ${ }^{7}$. Nous aurions donc bien

5. Les résultats de cet énorme chantier sont en cours de publication. Néanmoins, des synthèses ont été progressivement publiées, cf. BARDEL, Annie, "Archéologie ", dans SimON, Marc, L'abbaye de Landévennec..., op. cit., p. 199-209; "L'Abbaye Saint-Gwénolé de Landévennec ", Archéologie Médiévale, 21 (1991), p. 51-101; "Douze siècles à même le sol. Derniers acquis d'une recherche archéologique à Landévennec ", Chronique de Landévennec, $3^{\mathrm{e}}$ série, 4 (oct. 2000), p. 181-190; pour le haut Moyen Âge, voir la synthèse la plus récente : ID., "Les premiers bretons à Landévennec, $\mathrm{VI}^{\mathrm{e}}$-IX $\mathrm{X}^{\mathrm{e}}$ siècles ", dans GIOT, Pierre-Roland, GUIGON, Philippe et MERDRIGNAC, Bernard, Les premiers Bretons en Armorique, Rennes, PUR, 2003, p. 129-133.

6. Sur l'ensemble de ces textes ainsi que sur leurs dérivés médiévaux, la synthèse la plus récente est due à Poulin, Joseph-Claude, "Le Dossier de saint Guénolé de Landévennec ", Francia 23/1 (1996), p. 167-205. Ce dossier dispose de deux éditions simultanées : DE SMEDT, C. "Vita S. Uuinuualoei abbatis Landevenecensis, auctore Wurdestino, nunc primum integre edita " dans Analecta Bollandiana 8 (1888), p. 167-264 et LA BORDERIE, Arthur Le Moyne de, Le Cartulaire de Landévennec, Rennes, 1888.

7. Poulin, Joseph-Claude, "Les réécritures dans l'hagiographie bretonne (VIII$\mathrm{XII}^{\mathrm{e}}$ siècles) "dans Goullet, Monique et Heinzelmann, Martin (dir.), La Réécriture 
là un nouvel exemple tout à fait frappant de continuité, cette fois-ci dans la vie spirituelle de la communauté, et non plus seulement dans sa vie matérielle. Autre coïncidence, l'un de ces textes, la Grande Vie de Guénolé, utilise selon la pratique hagiographique classique la biographie de son fondateur pour retracer l'histoire primitive de son monastère. C'est dans ce contexte que Gurdisten propose de nombreuses descriptions du site, qui offrent donc au chercheur un autre angle d'approche dans l'étude de cet espace monastique.

Cette convergence factuelle et thématique ne peut manquer de poser la question d'un lien éventuel entre ces deux éléments, qu'ils aient tous les deux la même cause ou que l'un des aspects ait influencé l'autre. Pour éclaircir ce point et mieux pénétrer les arcanes des mentalités d'une communauté monastique au premier siècle bénédictin en Bretagne, il est nécessaire de relire avec attention l'œuvre hagiographique de Gurdisten, afin d'en dégager les implications et mieux en comprendre l'impact.

\section{"Locus amoenissimus " : l'invention d'un paysage?}

Le premier, et de prime abord le plus évident des niveaux de lecture de cette œuvre essentielle est la simple description littéraire de la topographie du site de Landévennec, morcelée au début du deuxième livre de la Grande Vie de Guénolé.

Suivant un cliché hagiographique, les moines s'établissent en un locus amoenissimus, qui fait l'objet de longues descriptions dont il faut avant tout souligner la relative originalité, du moins par leur caractère prolixe, dans l'hagiographie bretonne contemporaine. Si le paysage n'est pas absent des autres textes, il n'apparaît que par petites touches : telle forêt est "vastissima ${ }^{8}$ ", refuge des bêtes sauvages; un lieu est " secretus ", véritable désert, ou au contraire fertile, propice à l'agriculture. On mentionne ici un arbre fruitier, là une hauteur. Les sources sont "lucidissimae ", une grotte, refuge d'un dragon, est "impenetrabile " ". Quant aux sites des monastères, ils sont qualifiés de manière souvent très expéditive par une série d'adjectifs valorisants, mais qui ne nous disent finalement pas grand chose de la topographie du lieu. La seule chose que nous dit par exemple l'auteur anonyme de la Vita Ia Samsonis concernant l'aspect du monastère d'Iltut, est qu'il était "magnifico ${ }^{10}$ "; l'hagiographe reprend à nouveau cette expression pour désigner la fondation samsonienne de Pentale ${ }^{11}$.

hagiographique dans l'Occident Médiéval. Transformations formelles et idéologiques, Ostfildern, Beihefte der Francia band 58, 2003, p. 145-194.

8. Vita Ia Samsonis 1, 26, Flobert, Pierre (éd.), La Vie ancienne de saint Samson de Dol, Paris, CNRS éditions, ci-dessous : 1 VS.

9. 1 VS 1,50 .

10. $1 V S 1,7$.

11. 1 VS 1,59 . 
Autre remarque méthodologique, en quels termes serait-il correct de raisonner pour appréhender la représentation de l'espace chez Gurdisten? Un mot vient naturellement à l'esprit, celui de " paysage ", tant il est lié à la notion de rendu pictural ou littéraire des traits physiques d'un environnement donné. Mais son emploi à une si haute époque pose problème. Si l'on se place du point de vue des géographes de l'école vidalienne, le paysage est formé d'un ensemble de traits morphologiques naturels, c'est une notion objective. Il n'y a donc aucun problème à en faire usage pour étudier la prose d'un auteur du IX ${ }^{\mathrm{e}}$ siècle.

En revanche, si l'on adopte une démarche esthétisante, la société médiévale ne remplit pas les quatre critères posés par Augustin Berque pour la qualifier de " paysagère ". En effet, il y manquerait le mot même de paysage $^{12}$, quand bien même des auteurs y développeraient un certain sens de la beauté et du charmant. André Roger, qui suit sur ce point Berque, parle ainsi de "la cécité médiévale ${ }^{13}$ ". Ils ne reconnaissent une timide " invention du paysage " que tardivement, au XIV ${ }^{\mathrm{e}}$ siècle, sans que le mot lui-même soit apparu ${ }^{14}$. À ce titre la société médiévale serait " proto-paysagère " et guère plus, elle poserait un regard limité, " au bord du chemin " et " au ras du sol ", sur le pays et non sur le paysage ${ }^{15}$.

L'historien se positionne en fait entre ces deux extrêmes. Il n'attache pas l'importance des historiens de l'art à la notion d' "artialisation ". Le paysage n'est pas non plus ce concept objectif, donné a priori, des vidaliens, mais est bien issu d'une construction, d'une interaction entre le regard d'une société et son environnement au sens large. Le rapport au paysage est pour lui bien sûr objectif. L'homme le travaille, le modifie en fonction de ses besoins, la plupart du temps pour assurer sa subsistance. L'historien peut donc essayer de l'appréhender comme le témoignage d'une action de l'homme, un objet " construit ${ }^{16}$ ", au même titre qu'une cathédrale ou un château. Les études d'histoire rurale s'appuient ainsi largement sur ce premier rapport.

Mais la relation à l'espace de l'homme médiéval comporte aussi une dimension subjective évidente, qui découle sans aucun doute du rapport de dépendance matérielle entre lui et son environnement. Le voyageur et le pêcheur sont soumis aux caprices des flots? Les littérateurs médiévaux les décriront en des termes virgiliens épiques, effrayants. Le paysan et la société entière dépendent de la production agricole? Une terre fertile sera alors pensée comme un "novus paradisus ". À l'inverse, les espaces arides seront lieux d'épreuves spirituelles, ce désert néo-testamentaire où Jésus

12. RoGer, Alain, Court traité du paysage, Paris, Gallimard, NRF, coll. « Bibliothèque des sciences humaines", 1997, p. 48.

13. Ibid., p. 58.

14. Ibid., p. 65 .

15. Ibid., p. 59.

16. Antorne, Annie, Le Paysage de l'historien, Archéologie des bocages de l'Ouest de la France à l'époque moderne, Rennes, PUR, 2000. 
par trois fois repoussa les tentations du démon. La topographie médiévale étant bien sûr différente de celle du Moyen Orient biblique, c'est souvent la forêt, espace pour une large part indompté, qui prendra le relais dans l'imaginaire médiéval, comme l'analysait Jacques Le Goff dans une étude désormais classique ${ }^{17}$.

On le sent bien, le point commun entre ces exemples est une manière de ressentir l'espace, de percevoir le naturel et ses manifestations à travers l'application d'une grille de lecture préexistante, qu'il s'agisse de clichés littéraires hérités des auteurs classiques tant admirés ou des valeurs morales qui y sont attachées dans la Bible ou chez les Pères de l'Église. Par conséquent, nous sommes bien dans un rapport mixte qui à mon sens autorise l'emploi et l'étude du concept de paysage pour la période médiévale.

Or, il ne fait aucun doute que ce paysage matériel tout empli de considérations esthétiques et morales est présent chez Gurdisten, de manière même plus importante semble-t-il que chez les autres hagiographes. L'abbé de Landévennec fait tout d'abord preuve d'un sens remarquable de la perception et de la représentation précise de la topographie. Il s'essaye par exemple à l'évaluation des distances : selon, lui, le bras de rivière séparant l'île de Tibidi où s'établit dans un premier temps la jeune communauté, de la presqu'île de Landévennec mesure environ deux milles ${ }^{18}$. S'il ne tente pas d'évaluer les dimensions du vallon au creux duquel se niche son monastère, il en décrit la configuration générale en ayant essentiellement recours, et ce de manière insistante, au filage de trois métaphores : la fronde, l'arc tendu et les cornes. Toutes trois rendent effectivement bien compte de la situation du vallon de Landévennec, adossé à une hauteur qui l'entoure de trois côtés. Gurmonoc, disciple de Gurdisten, reprendra d'ailleurs la première pour évoquer dans sa vie de Paul Aurélien le site de l'oppidum Pauli, aujourd'hui Saint-Pol-de-Léon ${ }^{19}$.

En outre le moine de Landévennec ne se montre pas imperméable à un certain sens du charmant, comme en témoigne cette description du ruisseau qui au IX ${ }^{\mathrm{e}}$ siècle encore courait au milieu du site :

"En effet (ce lieu) est arrosé par un ruisseau puisant sa source en amont, et de ce bouillonnement scintillant jaillit nouvellement des profondeurs de la terre une source très limpide, le léger murmure de la course de ses eaux invitant à un très doux sommeil l'homme las après le travail ${ }^{20}$."

17. LE GoFf, Jacques, L'Imaginaire médiéval, Paris, Gallimard, NRF, 1984, p. 64 sqq.

18. Vita Amplior Winwaloei auctore Gurdestino II, 3, De SMEDT, C. (éd.), "Vita S. Uuinuualoei...", art. cit., p. 217. Ci dessous : VAW.

19. « in modum arcus cum maxime tenditur per circuitum ejus undique deflexo ", Vita Sancti Pauli Aureliani, 15, Cuissard, Charles (éd.), " Une vie de saint Paul en Léon d'après un manuscrit de Fleury-sur-Loire ", Revue Celtique 5 (1883), p. 442. Ci-dessous : VPA.

20. «nam a superioris fontis rivuli superfundentis haustu irriguus et a quodam lucidissimi fontis bucliamine gemmatus ex profundo terrae noviter erupto cum leni murmure decurrentium aquarum suavissimum fessis post laborem suadentium soporem " VAW II, 26. 
Que cette description fasse écho à des clichés littéraires antiques, virgiliens en particulier, importe finalement peu. Ce qui compte, c'est que l'imagination de l'auteur ait pu en être frappée et durablement impressionnée, au moins suffisamment pour se les réapproprier.

Mais Gurdisten ne se limite pas dans ses descriptions à un simple appel au pathos, loin de là. Son approche de l'espace semble en fait bien structurée, et aux éléments strictement topographiques, presque laïcs oserais-je dire, succède un discours infiniment plus subjectif. La transition se fait, sans trop de surprises, par une intrusion du divin : « Dieu, dis-je, a aménagé ce lieu pour ces serviteurs ${ }^{21}$. " Au-delà de ce tournant rhétorique, chaque élément est une preuve supplémentaire du discours de Gurdisten. La flore, par exemple, est intégrée dans une réflexion chrétienne. La rédemption tout d'abord : " Le jardin est orné de fleurs de toutes les couleurs, et l'on y peut cueillir non seulement des fruits terrestres, mais surtout des fruits célestes ${ }^{22}$. " Quelques chapitres plus loin, son odeur est associée à l'odeur de sainteté :

"Non seulement [ce lieu] est planté d'arbres fruitiers de toutes sortes et empli de l'agréable odeur de légumes extraordinaires mais, ce qui est plus important encore, il est agrémenté du parfum des saints corps y reposant, qui en raison de leur multitude ne pourront jamais être dénombrés par quiconque $^{23}$."

Le ruisseau évoqué plus haut perd également de son caractère anodin sous la plume de Gurdisten, puisqu'il lui donne l'occasion d'un rapprochement avec le jardin d'Éden : « Avec raison, on appelait [ce lieu] un nouveau paradis, au milieu duquel s'écoule un large ruisseau ${ }^{24}$. "C'est aussi l'une des caractéristiques principales de l'Éden : « Un fleuve sortait d'Éden pour irriguer le jardin; de là il se partageait pour former quatre bras ${ }^{25}$. " C'est en fait une constante de la mentalité médiévale que de se représenter l'Éden comme un lieu de repos calme et rafraîchissant.

Il est donc patent que nous n'avons pas chez Gurdisten un regard au " raz du chemin ", mais bien une perception complète de l'espace dans ses dimensions physiques et spirituelles. Certes, d'autres œuvres présentent en la matière des points de contact avec les descriptions de Landévennec. Au IX ${ }^{\mathrm{e}}$ siècle, les Gesta Sanctorum Rotonensium décrivent le site du monastère de Saint-Sauveur de Redon dans des termes assez proches de ceux qui naissent sous la plume de Gurdisten ${ }^{26}$. Néanmoins, leur démarquage pro-

21. "locum, inquam, a Deo servitoribus suis est praeparatus ", VAW II, 5.

22. " hortus omnigeno florum colore decoratus, in quo non solum terrestres, sed caelestes potius adquiruntur fructus ", ibid.

23. « modo jam non solum arboreis omnigeni pomarii venustatibus plantantus et holerum mirificis odoribus repletus, sed, quod multum majus est, sanctorum fragrantia corporum illic pausantium decoratus, qui nequaquam prae multitudine ab aliquo possunt denumerari ", VAW II, 7.

24. "novus videtur paradisus jam veraciter dictus, ex quo jam in medio fons patet largissimus ", VAW, II, 7 p. 222.

25. Genèse 2,10.

26. Gesta Sanctorum Rotonensium I, 3, BrETt, Caroline (éd.), The Monks of Redon. Gesta Sanctorum Rotonensium and Vita sancti Conwoionis, The Boydell Press, 1989, p. 119. 
bable de la Vita Sancti Filiberti ${ }^{27}$, en diminue la portée. C'est ce même passage que condense au $\mathrm{XI}^{\mathrm{e}}$ siècle l'auteur de la Vita Conwoionis :

"Ce même lieu est si remarquable de par sa position naturelle qu'il surpasse en beauté tous les autres monastères de la Bretagne gauloise, ceint de montagnes semblables à des murailles dont la cîme se rapproche du ciel, sur une terre très agréable qui dispense la grâce de tous les délices ${ }^{28}$."

Les Miracula Maglorii prennent également la peine de souligner le caractère paradisiaque de l'environnement du monastère de Léhon, situé " au flanc d'une montagne sur la rive d'un fleuve, en terrain plat parmi les herbes odorantes, les pins et les arbres fruitiers ${ }^{29}$ ".

Mais de telles précisions demeurent en définitive assez rares, et aucune ne bénéficie d'un traitement aussi important que Landévennec. Gurdisten et, dans une moindre mesure, Gurmonoc avec sa description de l'oppidum Pauli, font donc tout de même exception dans une littérature hagiographique plus prompte à aller à l'essentiel, autrement dit le fait miraculeux et édifiant, ainsi que son commentaire homélitique. Le potentiel exégétique d'une description paradisiaque d'un lieu n'est certes pas nul puisqu'il a des implications morales tout à fait valorisantes pour une communauté, mais l'on ne sort guère du topos. Chez Gurdisten en revanche, ce cadre étriqué du locus amoenissimus est dépassé. L'environnement, mais parlons plutôt de paysage, fait partie intégrante de sa dialectique et lui offre son support, voire même sa structure.

\section{Dialectique de la mort...}

Il semble que la structure du discours de Gurdisten soit axée autour de trois mises en opposition géographiques qui tendent à démontrer la sainteté du site de Landévennec et à en présenter les caractères essentiels. La première lui oppose l'île de Tibidi, premier refuge de Guénolé et ses compagnons, décrite en des termes austères :

"Ce lieu rude, dis-je, ouvert à tous les vents, ceint de tous côtés par la mer et des rochers saillants, n'était digne d'aucune habitation humaine [...] mais parce que ce lieu indigne ne leur convenait absolument pas, non pas tant en raison de son infertilité que de la violence des vents et des orages, ils s'attristaient d'y être confinés ${ }^{30}$."

27. SmITH, Julia M. H., « Aedificatio sancti Loci : The making of a ninth-century holy place », dans Topographies of medieval power in the Early Middle Ages, Jong, Mayke de, THEuws, Frans, RHIJN, Carine van et Brill, Leiden-Boston-Köln (éd.), 2001, p. 383 et note 86.

28. "Ipse vero locus adeo naturali positione insignis habetur ut amoenitate sua ceteris Britanniae Gallicanae locis praestet, montibusque proceritate sua polo vicinis quasi quibusdam moeniis ambiatur, et omnium deliciarum gratiam proferat gratissima tellus ", Vita Conwoionis 3, BRETT, C. (éd.), The Monks of Redon..., op. cit., p. 228.

29. Miracula Maglorii 8, 23, LA BORDERIE, A. de (éd.), « Les Miracles de saint Magloire : texte latin et français avec notes et commentaires historiques " dans Mélanges de la Société d'Émulation des Côtes-du-Nord, 4 (1891), p. 230-256.

30. «Locus, inquam, asperrimus, ad omnem ventum porrectus, mari undique paene et acutis rupibus praecinctus, nulli humanae habitationi dignus. [...] sed quia nullo modo indignus 
Nous avons donc là une opposition topographique et climatique claire à laquelle s'ajoute, ce qui est sans doute encore plus important, le problème de la sanctification de la terre :

" Dieu ne voulut pas consacrer un tel lieu par l'ensevelissement de la dépouille de l'un des disciples de saint Guénolé, car il semblait préférable que tous les membres soient rassemblés à l'endroit où leur tête reposerait un jour ${ }^{31}$."

De manière significative, le site de Landévennec est mentionné pour la première fois juste après cette remarque, site que les moines observent déjà avec amour du haut d'une colline ${ }^{32}$. Par conséquent, un lieu " où reposent à présent les os (de saint Guénolé) ", autrement dit la " tête " de la communauté " et de ses compagnons ${ }^{33}$ ", les "membres ", est consacré par Dieu. Cette notion est centrale, puisque la seconde opposition repose elle aussi finalement sur la question funéraire.

L'installation des moines sur l'autre rive de l'Aulne ne s'est en effet pas faite en une seule fois. Gurdisten rapporte ainsi cette fameuse légende : le site primitif de l'abbaye se trouvait situé sous un trou dans le ciel, par lequel les anges montaient et descendaient ${ }^{34}$. Quant aux moines, bien que vieillissants, ils ne pouvaient mourir ${ }^{35}$. Guénolé obtient alors par la prière l'autorisation de déplacer le monastère " à un jet de pierre " dans la direction du levant, à l'emplacement exact du site actuel. Or, Gurdisten insiste à nouveau : cet endroit est " rendu plus noble, par l'action de Dieu ${ }^{36}$ " que le site primitif, car les moines peuvent y mourir. Il faut donc les inhumer, et c'est bien la présence de ces saints corps qui donne toute sa sacralité au lieu. Il en évoque même le lieu précis :

" Il a été rapporté par de très droits et très vénérables anciens que [Guénolé] enterra ensemble de ses propres mains de nombreux membres de sa communauté qui l'avaient précédé au Royaume céleste dans un cimetière appelé les Reliques des Saints ${ }^{37}$."

conveniebat eis locus, non tam propter infertilitatem quam ventorum procellarumque immnanitatem, retardati contristabantur", VAW II, 3.

31. " quia talem locum noluit Deus vel unius corpusculi discipulorum sancti Winvaloei sepultura consecrari, sed ubi eorum caput quandoque quiesceret, ibi decentius cuncta illius membra collocarentur assumpta ", VAW II, 3, p. 216.

32. Ibid., p. 216-217.

33. "ille locus [...] in quo nunc ossa ejus cum sociis pausant ", VAW II, 26, p. 241.

34. " et dixissent apertum esse tantum caeli spatium supra illum locum in caelo quantum terrae occuparat, et angelorum illic sancto Wingualoeo demonstrante visione fruitos fuisse ascendentium et descendentium in similitudine visionis Jacob patriarchae ", Ibid.

35. "Sed tamen hoc ne unquam oblivioni tradatur, quod nemo in eodem loco potuit mori ", Ibid., p. 244.

36. "Qui ibidem locus, Deo agente, nobilior factum est alio jam dicto ", VAW II, 26, p. 242.

37. "Senes etenim maximi et justissimi ferebant quod multi de illa congregatione praecessissent illum ad regna caelestia, de manu ejus propria in uno coemiterio, quod dicunt Reliquias sanctorum, consepulti ", VAW II, 28, p. 246. 
À plusieurs reprises dans ce second livre, Gurdisten ne manque pas de rappeler à ses lecteurs, ses frères, leur proximité avec ces saints corps ${ }^{38}$.

Cette dialectique ne vaut d'ailleurs pas seulement au plan strictement local. L'ensemble de ces descriptions contrastent visiblement avec le tableau extrêmement négatif qui nous est dressé de la description physique et morale de la Bretagne insulaire dans le livre I, tableau qui doit par ailleurs beaucoup au De Excidio Britanniae de Gildas, sage auquel Gurdisten fait nommément référence : "Que celui qui voudrait en savoir plus lise saint Gildas ${ }^{39}$. " La lecture de cet ouvrage capital du " dernier des romains " est également attestée chez Gurmonoc, qui le connaît sous le titre De Ormesta Britanniae $^{40}$. Gurdisten reprend par exemple l'épisode de la "pestilentia " déjà rapporté par Gildas, mais il précise que les gens sont empilés " absque numero et absque sepultura ", ce qui est une abomination pour des chrétiens. Si les corps saints qui embaument l'air à Landévennec " ne peuvent être dénombrés ${ }^{41}$ ", ils bénéficient en revanche d'une sépulture chrétienne.

De plus, dans le cas de la Grande Bretagne, ces morts sont présentées comme un châtiment divin, apportant la malédiction sur la terre, tandis que le trépas des saints de Landévennec est présenté comme une récompense, permettant l'accession au Royaume céleste. Enfin, si la terre de Bretagne insulaire est, tout comme celle de Landévennec, fertile en " froment, lait et miel ${ }^{42}$ ", Landévennec se distingue par le fait que les " fruits terrestres ", comme on l'a vu, y avoisinent les " fruits célestes ", tandis qu'en Bretagne Insulaire, "l'abondance allait être à la source de ses maux ${ }^{43}$ ".

Comme le suggère déjà cette métaphore et comme le font remarquer le chanoine Du Cleuziou ${ }^{44}$ et Bernard Merdrignac à sa suite, cette opposition structure le développement d'un discours eschatologique, centré sur une " spéculation sur le mystère pascal qui entoure le récit de la fondation de Landévennec ${ }^{45}$ ", qui calque ses grandes étapes suivant une typologie de l'Exode. La Bretagne insulaire est l'Égypte, Landévennec la Terre Promise. La fondation du monastère ouvre un âge d'or, un millenium. Pour Bernard Merdrignac, cette coloration eschatologique serait le fait de la version primitive utilisée par Gurdisten, qui s'inscrirait en plein cœur des débats sur

38. « in eo, quo ossa ejus sacra nunc condita sunt », VAW II, 1, p. 212 et " ubi nunc sacrum corpus ejus cum innumerabilis sanctorum sociorum requiescit corporibus ", VAW, II, 4, p. 217

39. "qui haec plenius scire voluerit, legat sanctum Gildam ", ibid., I, 1.

40. VPA 3

41. VAW II, 7, p. 222.

42. " frumenti scilicet mellis lactisque simul fertilissima ", VAW I, 1, p. 174.

43. " abundantia rerum causa fuit malorum ", ibid., p. 175.

44. Cleuziou, Jacques Raison du, « La Fondation de Landévennec », Mélanges de la Société d'Émulation des Côtes-du-Nord 114 (1986), p. 10.

45. MERDRIGNAC, Bernard, « Temps historique, temps liturgique et temps archéologique : la légende des Immortels de Landévennec ", communication à la journée d'étude de mai 2002 de la Maison de la Recherche en Sciences Sociales de l'Université de Rennes 2, (à paraître). Je remercie le professeur Merdrignac de m'en avoir aimablement fourni le texte. 
le mode de calcul de la date de Pâques qui agitèrent la Chrétienté au très haut Moyen Âge ${ }^{46}$.

Il ne me semble pas nécessaire, pour l'instant, de s'aventurer aussi loin dans des supputations sur une Vita Prima sur laquelle on sait très peu de choses, et dont on n'a pas encore prouvé, à mon avis, l'existence sous une forme aussi cohérente que celle que lui suppose cette réflexion. Je ne vois en fait aucune objection décisive à ce que la paternité de cette organisation de la Vita soit retirée à Gurdisten, car elle me semble tout à fait correspondre à l'esprit de passages dont l'attribution à l'abbé de Landévennec me paraît difficilement contestable. C'est dans un contexte agité, celui de la seconde moitié du IX ${ }^{\mathrm{e}}$ siècle, que Gurdisten se livre à ces considérations sur le passé de son abbaye, passé idéalisé à l'extrême en comparaison avec les difficultés que doit affronter le regnum breton à cette époque.

Si le temps de la communauté de Guénolé est effectivement considéré comme un âge d'or de la Bretagne Armorique, celui-ci peut, de manière crédible, paraître révolu à Gurdisten. Mais lui qui vit là où vécut Guénolé, lui qui marche avec ses frères dans le même paysage, qui peut contempler Tibidi depuis la rive devant son monastère, tire des exemples du passé une conviction certaine d'un retour possible à l'ordre et à l'harmonie perdue. Cet acte de foi trouve son expression la plus forte dans les chapitres versifiés du livre II, qui sont une véritable ode à la Cornouaille, dont la destinée lui paraît indissociable de celle de Landévennec. L'abbaye est décidément l'endroit ou chacun est plus proche de son salut, individuellement en tant qu'homme, collectivement en tant que moine ou cornouaillais ${ }^{47}$.

Cela dit, la centralité de la question funéraire et l'importance symbolique qui y est attachée se traduisent aussi de manière concrète, puisque le cimetière primitif, accolé au chevet de la basilique, est en usage de manière continue jusqu'au XIII ${ }^{\mathrm{e}}$ siècle (fig. 1). Sa mise en scène constante dans le récit de Gurdisten n'est d'ailleurs pas sans évoquer un classique du monachisme carolingien, le célèbre plan idéal de Saint-Gall. Rappelonsnous ce trait de la description de Landévennec :

"Non seulement [ce lieu] est planté d'arbres fruitiers de toutes sortes et empli de l'agréable odeur de légumes extraordinaires mais, ce qui est plus important encore, il était agrémenté du parfum des saints corps y reposant, qui en raison de leur multitude ne pourront jamais être dénombrés par quiconque $^{48}$."

46. Ibid.; Dictionnaire d'Archéologie Chrétienne et de Liturgie, vol. XIII/2, s. a. " Pâques " col. $1550 \mathrm{sq}$.

47. Cf. Cleuziou, Jacques Raison du, " La Fondation de Landévennec ", art. cit., p. 9 et n. 14.; MERDRIGNAC, Bernard, « La Vie quotidienne dans les monastères bretons du haut Moyen Âge... ", art. cit., p. 32.

48. « modo jam non solum arboreis omnigeni pomarii venustatibus plantatus et holerum mirificis odoribus repletus, sed, quod multum majus est, sanctorum fragrantia corporum illic pausantium decoratus, qui nequaquam prae multitudine ab aliquo possunt denumerari ", VAW II, 7. 


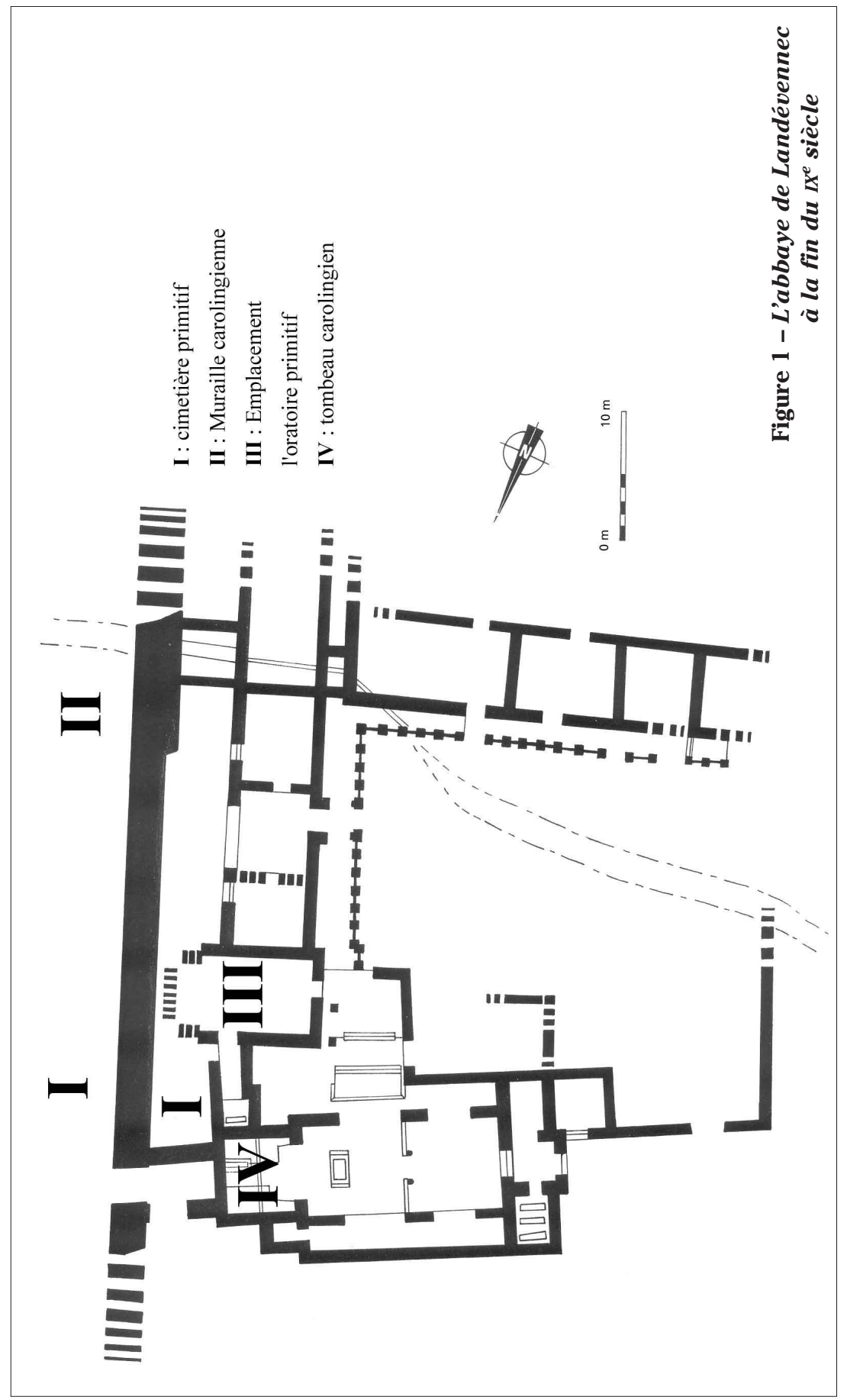


Gurdisten y associe implicitement l'ensemble verger et jardin avec le cimetière. Or, cette association rappelle fortement l'imbrication du cimetière et du verger dans le plan idéal de l'abbaye de Saint-Gall ${ }^{49}$. Comme l'écrit Carol Heitz de fort belle manière : "Quatorze arbres y puisent leur sève dans la terre des tombes des frères ${ }^{50}$. " La teneur eschatologique qui conditionne cette disposition est capitale. Le Plan de Saint-Gall mentionne ainsi que trône au milieu du verger " l'arbre du Salut - la Croix - chargé des fruits de l'Éternité ${ }^{51}$ ", à Landévennec, comme en écho, c'est dans ce jardin, sous les arbres et non loin des corps saints, que l'on peut accéder aux " fruits célestes " que nous avons déjà évoqués.

Cependant, nous sommes là dans la conceptualisation d'un rapport à la tradition par l'entremise des morts, mais l'archéologie permet de fournir également un éclairage beaucoup plus nuancé. En effet, au siècle même de Gurdisten, le rapport aux saints de Landévennec est beaucoup plus pragmatique. Si le fameux cimetière, les "Reliques des saints", est bien en place et en usage jusqu'à la fin du Moyen Âge, la muraille édifiée au IX ${ }^{\mathrm{e}}$ siècle, certainement avant la rédaction de la Vita Winwaloei d'ailleurs puisque Gurdisten la mentionne, a empiété sur sa partie orientale. Les fouilles ont ainsi mis à jour des tombes littéralement sectionnées par les fondations de la muraille, dont les corps ont été cependant maintenus en place. Ceux dont la tête était placée à l'ouest sont amputés au niveau des fémurs, tandis que les moines ont malgré tout pris soin de collecter les crânes des squelettes orientés pour les placer entre leurs jambes (fig. 2) ${ }^{52}$.

Cette prise de liberté avec les dépouilles ne choque pas réellement au regard des pratiques connues de réutilisation de tombes ou de sarcophages dans les cimetières tout au long du Moyen Âge. Cependant, ces " profanations " sont souvent le résultat de contraintes extérieures naturelles ou sociales, comme en témoignent les textes littéraires ou canoniques. L'érosion d'un tertre ou l'abandon relatif d'un site funéraire en fait disparaître par exemple les marques extérieures, et c'est un paysan qui en mettant en culture ces parcelles endommage les sépultures. Une " tribulation ", famine ou épidémie, augmente le nombre de décès, donc la pression sur l'espace funéraire forcément limité à partir du moment où il est clos, intra muros dans les cités ou ad sanctos dans les monastères, et la population ensevelit les dépouilles récentes dans la même fosse ou le même sarcophage, faute de place. Ces désagréments arrivent même, comble de l'horreur, aux saints eux-mêmes ${ }^{53}$. À d'autres périodes, la législation canonique

49. Cette analogie m'a été suggérée par Bernard Merdrignac. Je l'en remercie.

50. Heitz, Carol, L'architecture religieuse carolingienne, Paris, Picard, p. 170.

51. Ibid.

52. BARDEL, Annie et PERENNEc, Ronan, "Abbaye de Landévennec : évolution du contexte funéraire depuis le haut Moyen Âge ", dans Inhumation et édifices religieux au Moyen Âge entre Loire et Seine, actes du séminaire du CRAHM du 18 octobre 2003 à Caen - à paraître. Je remercie Annie Bardel et Ronan Pérennec de m'avoir permis d'utiliser ces données.

53. PIETRI, Luce, "Les Sépultures privilégiées en Gaule d'après les sources littéraires ", dans L'Inhumation privilégiée du IV $v^{\mathrm{e}} a u$ VII siècle en Occident, Actes du colloque tenu à 
Landévennec au IX ${ }^{\mathrm{e}}$ siècle

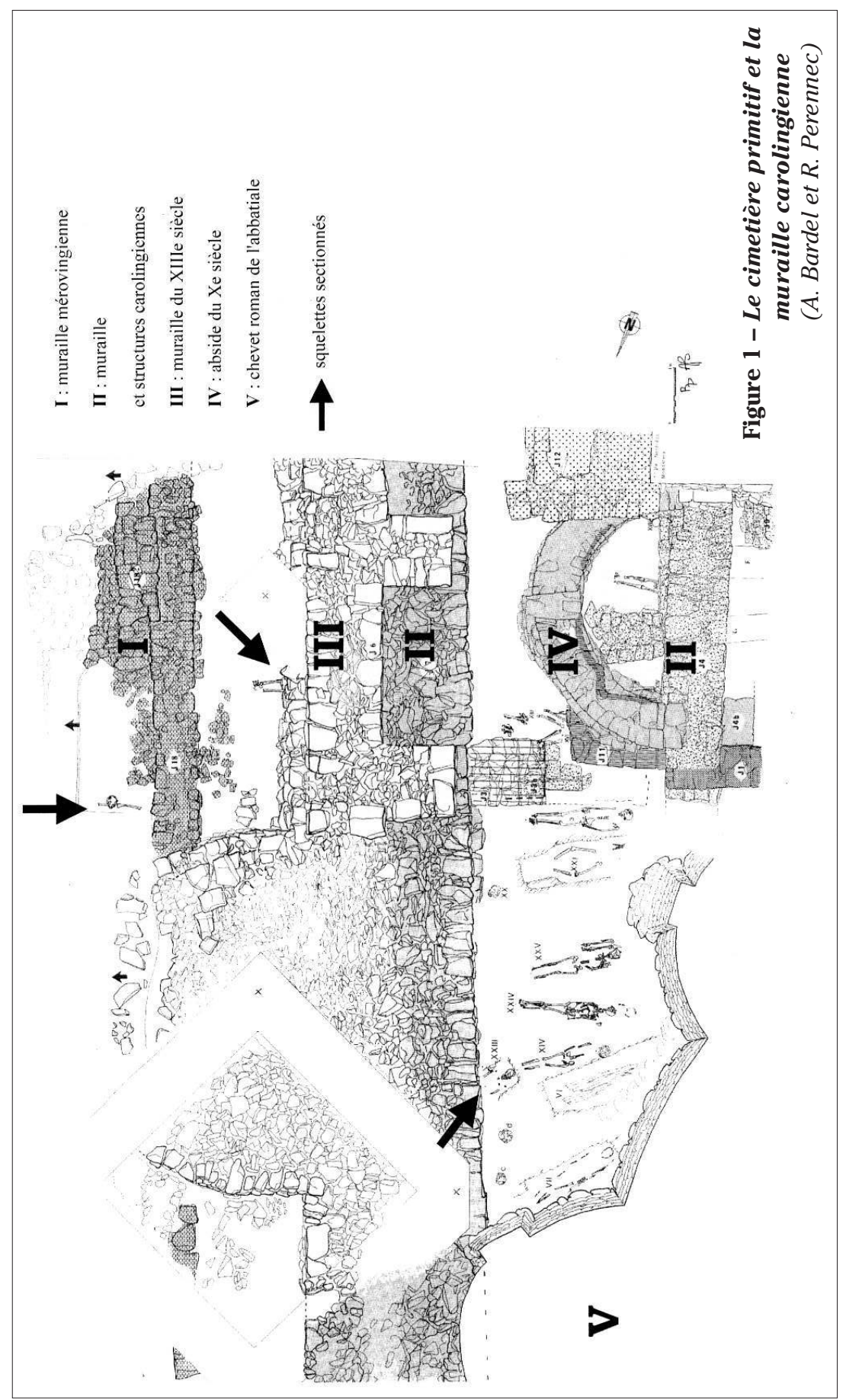


rappelle certaines règles concernant les lieux acceptables d'inhumation ${ }^{54}$, et prescrit le déplacement des corps y contrevenant. Les prélats carolingiens rappelèrent par exemple à maintes reprises l'interdiction d'enterrer dans les églises, et prescrivent entre autres un renfouissement plus profond pour en faire disparaître les traces ${ }^{55}$.

Nous avons là affaire à des accidents, des hasards ou des réorganisations destinées à rétablir un ordre religieux ou social correct. Cela pourrait bien entendu être aussi le cas à Landévennec, mais la muraille est élevée en plein milieu d'une parcelle reconnue de tout temps pour être le cimetière communautaire. Elle est donc $a$ priori bien délimitée, notamment par la clôture monastique mérovingienne, située au-delà de la fortification carolingienne. Ce n'est donc pas tant le traitement infligé aux dépouilles qui surprend, car nous en avons de nombreux exemples par ailleurs pour le Moyen Âge ${ }^{56}$, mais bien plutôt l'empiètement sur un espace qui occupe une place aussi centrale dans l'organisation matérielle du monastère et qui constitue un véritable socle de l'identité communautaire. On comprend ainsi mal que les moines ne se soient pas contentés d'araser la clôture mérovingienne pour reconstruire par-dessus, comme ils l'ont fait constamment partout ailleurs. Car enfin ces corps sont censés être ceux des saints de Landévennec, ces compagnons de Guénolé mis en terre, selon Gurdisten, par le saint lui-même. Cette curiosité archéologique et culturelle met en avant le décalage, ici tout à fait saisissant, entre un discours officiel et son application.

La Grande de vie de Guénolé est donc manifestement construite autour d'un discours sur l'espace qui se résout dans la mise en exergue de la présence des corps saints à Landévennec. Ils sacralisent la monachia et introduisent une épaisseur historique notable, car ils témoignent d'une époque révolue tout en assurant la transition et la continuité avec celle-ci. Cet espace littéralement en trois dimensions, physique, temporelle et spirituelle est caractéristique d'un système de pensée monastique promu au sein de la communauté de Landévennec, qui vise à refonder l'identité de la communauté à la fois dans le souci de la tradition et de la mise en conformité avec les standards carolingiens, notamment bénédictins. Les reliques, et pas seulement celles du saint fondateur, mais aussi les corps de sa communauté, sont bien au centre de la vie matérielle et spirituelle de la communauté.

Créteil les 16-18 mars 1984, Duval, Yvette et Picard, Jean-Charles (éd.), Paris, De Boccard, 1986, p. 134-136.

54. HeucLIN, Jean, " La Mort de l'abbé : un modèle de vie chrétienne ", dans Moines et moniales faces à la mort. Actes du colloque de Lille (2-4 octobre 1992), Paris-Lille, CAHMER-CREDHIR, Histoire Médiévale et archéologie 6, 1993, p. 27.

55. TREFFORT, Cécile, L'Église carolingienne et la mort, Lyon, Presses Universitaires, 1996, p. 138.

56. Cf. par exemple RaCinEt, Philippe, "Pratiques funéraires dans deux monastères picards ", Moines et moniales faces à la mort, op. cit., p. 251 et doc. 16 et 18, p. 260-261. 
Ceci ne constitue pas véritablement une révélation fracassante : le rôle des reliques dans les mentalités médiévales est bien connu depuis longtemps. Mais deux faits inhabituels sont à souligner. D'une part, Gurdisten ne leur accorde pas, ou du moins ne mentionne que très succinctement l'un des pouvoirs qui leur est traditionnellement attaché, la thaumaturgie. Ainsi peut-on lire en toute fin, à propos du corps de Guénolé : "Par lui toutes les choses indispensables sont fournies à leur requête et de nombreux miracles ont été accomplis ${ }^{57}$. " La Grande Vie est certainement destinée dans un premier temps au public restreint des moines de Landévennec, qui n'ont, en toute logique, pas besoin d'être convaincus de la virtus de leur saint. Le premier abrégé de la Grande Vie, composé par Gurdisten lui-même sous la forme d'une homélie, remplit sans doute cette fonction plus spécifiquement liturgique liée à l'incitation au pèlerinage. Mais si elle fait état de la fête de la translation du corps de Guénolé de " la petite " - en fait l'oratoire primitif, faisant désormais office de sacristie "vers la grande église ${ }^{58}$ ", autrement dit la basilique carolingienne, on ne lit aucune mention, même générale, de miracles accomplis par la sainte dépouille.

En comparaison, d'autres vitae n'hésitent pas à insister plus clairement sur le rappel de la virtus du corps du saint patron. Selon Gurmonoc, Paul Aurélien se préoccupait déjà de son vivant de l'attractivité future de son corps, et sa vita s'achève entre autres sur ce rappel classique : " Et là, par le mérite du saint, sont accordés de nombreux bienfaits à tous ceux qui invoquent Dieu en vérité ${ }^{59}$. " L'anonyme de la Vita Ia Samsonis dit de son saint : "Nous avons parmi nous le signe de sa béatitude, bien entendu par les miracles que Dieu accomplit quotidiennement par son entremise. Amen $^{60}$. " Et effectivement, il entame à la suite un second livre entièrement dédié à la gloire posthume de Samson. Le diacre d'Alet Bili, rédigeant vers 870 la deuxième vie de saint Malo, retient un plan fort proche. Les deux derniers chapitres du livre I sont consacrés à la gloire posthume de saint Malo $^{61}$, tandis que le second livre développe le sujet et narre en outre les tribulations des reliques du saint évêque.

Gurdisten se démarque donc sensiblement de l'hagiographie épiscopale du haut Moyen Âge en ne développant explicitement que les aspects spirituels de la biographie de Guénolé. J'écris " explicitement " puisque ces textes s'adressent à un public médiéval qui résout de lui-même l'équation qui lie miracles posthumes et présence des reliques, sans qu'il soit forcément besoin de l'exprimer en toutes lettres. Il est également intéressant de

57. " per quem petitum omnia illis necessaria praestarentur, et plurimae virtutes agerentur ", VAW II, 29 p. 249.

58. Omelia auctore Wurdestino 12, éd. LA BORDERIE, Arthur Le Moyne de, Le Cartulaire de Landévennec, op. cit., p. 135.

59. VPA 21.

60. 1VS I, 61

61. Vita secunda sancti Machutis auctore Bili I, 88-89, LE Duc, Gwenaël (éd.), Vie de saint Malo, évêque d'Alet, Dossiers du CeRAA, B - 1979, Saint-Malo, 1979. 
noter que Gurdisten ne se préoccupe par ailleurs guère des aspects matériels, principalement fonciers, de la fondation de l'abbaye, questions qui sont pourtant des topoi d'une littérature hagiographique dont l'un des soucis est souvent de garantir les droits ou de soutenir les prétentions séculières de la communauté dont elle est issue.

D'autre part, ce qui est remarquable ici et que nous avons essayé de mettre en valeur par ce « dépeçage » littéraire du récit de Gurdisten, est la complexité rhétorique d'une œuvre par ailleurs d'une haute tenue littéraire. Sa mise en exergue au sein de la production hagiographique bretonne contemporaine souligne de manière étonnante l'un des caractères originaux de l'œuvre de Gurdisten, qui vise volontairement à charger émotionnellement un endroit, tout en l'inscrivant plus largement dans une histoire plus vaste, celle de la Cornouaille, qui recoupe le destin de la Bretagne et l'histoire chrétienne du Salut en général. Cette étude nous permet donc de sortir de l'analyse essentiellement pragmatique de la notion d'espace monastique qui prévaut souvent dans l'historiographie ${ }^{62}$, pour rentrer dans le domaine des représentations. On notera ainsi que même des cadres physiques et socio-culturels aussi rigides que l'espace et le temps sont en fait plus lâches qu'il n'y paraît et peuvent être réinvestis, d'une manière qui n'est pas sans évoquer le couple classique de l'exégèse, le sens littéral, qui est fixe, et le sens spirituel et métaphorique qui varie suivant les commentaires.

Le succès de cette démarche auprès de la communauté des frères de Landévennec est attesté dans le temps par la reprise, malgré son abord peu aisé, de ce texte au XI ${ }^{\mathrm{e}}$ siècle, fait que nous soulignions plus haut. Ceci illustre sans conteste le sentiment de continuité qui lie la communauté romane à ses prédécesseurs mérovingiens et carolingiens ${ }^{63}$. Véritable vecteur de la tradition, il contribue par-là même au renforcement d'une identité communautaire qu'il inscrit donc dans la durée, à une époque où de nombreux bouleversements politiques et religieux surviennent dans le monde chrétien occidental. Cette perception de l'espace est, en retour, à nouveau inscrite dans la pierre, puisqu'elle conditionne par ailleurs les choix formels lors des campagnes de réfection ou d'agrandissement, les

62. Cf. entre autres Heuclin, Jean, " Recherches sur la notion d'espace dans le monachisme gaulois primitif " dans Pratique et sacré dans les espaces monastiques au Moyen Âge et à l'époque moderne. Actes du colloque de Liessies-Maubeuge (26-27-28 septembre 1997), Histoire Médiévale et Archéologie, 9 (1998), p. 13-22; HERITY, Michael, « Les Premiers ermitages et monastères en Irlande (400-700) ", Cahiers de Civilisation Médiévale, 36 (1993), p. 219-261; MAGNOU-NORTIER, Élisabeth, "L'Espace monastique vu par Adalhard de Corbie, d'après ses statuts ", ibid., p. 51-70 et sa notion de "très grand monastère "; MERDRIGNAC, Bernard, "La vie quotidienne dans les monastères bretons du haut Moyen Âge, à partir des vitae carolingiennes " dans Landévennec et le monachisme breton dans le haut Moyen Âge..., art. cit., p. 19-44.

63. GUENEE, Bernard, Histoire et culture historique dans l'Occident médiéval, Paris, Aubier, collection historique, 1980, [rééd. 1991], p. 86-87, même s'il précise p. 86 : «Et nos historiens ne se contentent pas de décrire. Ils sentent ces monuments et ces ruines comme des vestiges du passé, d'un passé révolu et différent " (souligné par nous). 
moines conservant toujours la même orientation dictée par l'oratoire primitif, l'abbatiale étant donc légèrement désaxée par rapport au reste des bâtiments claustraux. Sans doute avons-nous, dans tous ces éléments de réflexion, l'un des secrets de cette stabilité à Landévennec qui nous étonnait tant en premier lieu.

\section{RESUME}

Les représentations originales de l'espace monastique dans l'hagiographie carolingienne de l'abbaye bretonne de Landévennec sont construites autour de la nature sanctificatrice des corps des premiers moines inhumés à l'intérieur de la clôture et semblent être au cour du projet idéologique de la communauté. Ses membres peuvent ainsi se situer de manière vivace dans une topographie et une temporalité chrétienne qui fondent une identité très forte.

\section{ABSTRACT}

Monastic space representations in carolingian hagiography produced at the breton abbey of Landévennec are built upon the sanctifying nature of the first monks holy bodies buried inside the cloister and appear to be a central point in the community ideological project. Its members are thus able to vividly locate themselves inside a christian topography and temporality, creating a very strong identity. 
